(C) 1989 IEEE. Personal use of this material is permitted. However, permission to reprint/republish this material for advertising or promotional purposes or for creating new collective works for resale or redistribution to servers or lists, or to reuse any copyrighted component of this work in other works must be obtained from the IEEE.

\title{
LONGITUDINAL COUPLING IMPEDANCE OF A SMALL OBSTACLE ${ }^{1}$
}

R.I. Gluckstern and $F$. Neri

Physics Department, University of Maryland, College Park, MD 20742

\section{Introduction}

In a recent paper ${ }^{2}$, the longitudinal impedance for a narrow pill-box with beam pipes was investigated analytically and numerically, in order to understand the structure of the impedance as a function of frequency in the region above the cutoff of the beam pipe. The field matching procedure described by Henke was used in the limit of a narrow pill-box and the impedance was expressed as sum which required a cutoff for convergence. In the present paper we start. with the integral equation for the field at the pipe radius ${ }^{4}$ and solve this equation rigorously in the case of a small obstacle of general shape. In this way we obtain a more accurate representation of the important broad resonance which dominates the behavior in the frequency range above the cutoff of the beam pipe.

\section{Analysis}

The starting point for the analysis is the integral equation obtained for the electric field in the obstacle at the plpe radtus. Specifically, we have

$$
\begin{gathered}
\int_{0}^{g} d z^{\prime} F\left(z^{\prime}\right)\left[K_{p}\left(\left|z^{\prime}-z\right|\right)+K_{c}\left(z^{\prime}, z\right)\right] \\
=j e^{-j k z}
\end{gathered}
$$

and

$$
\frac{z(k)}{z_{0}}=\frac{1}{k a^{2}} \int_{0}^{g} d z F(z) e^{j k z} .
$$

Here $k: / 2 \pi$ is the frequency, a is the pipe radius, $z_{0}=$ $120 \pi$ ohms is the impedance of free space, and the azimuthally symmetric cavity, of general shape in the $r, z$ plane, extends axially from $z=0$, to $z=g$ at the pipe radius $r=a$. Apart from a constant, $F(z)$ is the axial electric field for $r=a$ and $0<z<g$. The component of the kernel from the pipe field is

$$
K_{p}(|u|)=\frac{2 \pi j}{a} \sum_{s=1}^{\infty} \frac{e^{-j b_{s}|u| / a}}{b_{s}} .
$$

where

$$
b_{s}=\sqrt{k^{2} a^{2}-j_{s}^{2}}, \beta_{s}=\sqrt{j_{s}^{2}-k^{2} a^{2}} .
$$

Here $j_{s}$ is the sth zero of the Bessel function $J_{0}(x)$ and $b_{s}$ is to be replaced by $-j \beta_{s}$ when $j_{s}>k a$. The component of the kernel from the "cavity fields" is

$$
\mathrm{k}_{\mathrm{c}}\left(\mathrm{z}, \mathrm{z}^{\prime}\right)=4 \pi^{2} \sum_{\ell} \frac{\mathrm{h}_{\ell}(\mathrm{z}) \mathrm{h}_{\ell}\left(\mathrm{z}^{\prime}\right)}{\mathrm{k}^{2}-\mathrm{k}_{\ell}^{2}},
$$

where the orthonormal (azimuthally symnetric) modes of the cavity (with an imaginary metal wall at $r$ - a) are defined by

$$
\nabla \times \vec{e}_{\ell}=k_{\ell} \vec{h}_{\ell}, \nabla \times \vec{h}_{\ell}=k_{\ell} \vec{e}_{\ell}
$$

and where $h_{\ell}(z) \equiv\left[h_{\ell}(a, z)\right]_{\varphi}$ is the azimuthal component of the normalized magnetic field at $r=a$.

\section{Small Obstacle Solutions}

The "cavity kernel" in Eq. (2.5) requires a sum over the modes in the cavity. For a small cavity, this sum is dominated by the lowest mode for which lhe solution for the magnetic field is the constant field

$$
h_{o}(z)=\frac{1}{\sqrt{2 \pi a \Delta}}
$$

normalized so that $\int h_{0}^{2}(z) d v=1$ over the annular cavity of length $2 \pi a$ and cross sectional area $\Delta$. The frequency of this mode corresponds to $k_{0}=0$, so that

$$
K_{C}\left(z^{\prime}, z\right) \cong \frac{2 \pi}{k^{2} a \Delta}
$$

The "pipe kernel" in Eq. (2.3) is more difficult to evaluate for a small obstacle, since the sum in Eq. (2.3) does not converge if $|u|=\left|z^{\prime}-z\right|$ is set equal to zero. To proceed we add and subtract $k_{p}(g)$, which is independent of $z^{\prime}$ and $z$, to obtain

$$
k_{p}(|u|)=k_{p}(g)+\frac{2 \pi j}{a} \sum_{s=1}^{\infty} \frac{e^{-j b_{s}|u| / a}-e^{-j b_{s} g / a}}{b_{s}} .
$$

Since $|u| / a$ and g/a are very small, the major contributions to the sum in Eq (3.3) occur for large $s$, in which case we obtain

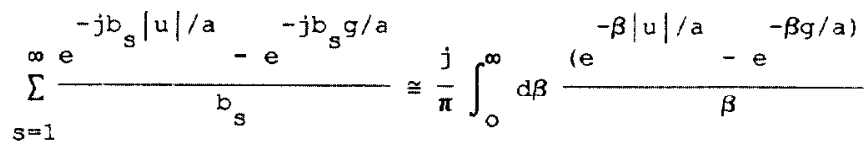




$$
=\frac{j}{\pi} \ln \frac{g}{|u|} .
$$

The integral equation for $F\left(z^{\prime}\right)$ in $E q$. (2.1) therefore becomes

$$
\int_{0}^{1} d x^{\prime} f\left(x^{\prime}\right)\left[x-\frac{2 j}{\pi} \ln \left|x^{\prime}-x\right|\right]=1
$$

where

$$
x^{\prime}=z^{\prime} / g, x=z / g, \pi g F\left(z^{\prime}\right)=a f\left(x^{\prime}\right)
$$

and

$$
K=-\frac{2 j}{k^{2} \Delta}+2 \sum_{s=1}^{\infty} \frac{e^{-j b_{s} g / a}}{b_{s}} .
$$

The solution of Eq. (3.5) can be expressed in terms of the solution of

$$
\int_{0}^{1} d x^{\prime} L\left(x^{\prime}\right) \ln \left|x^{\prime}-x\right|=1,0<x<1 .
$$

Specieically we find

$$
E\left(x^{\prime}\right)-\frac{L\left(x^{\prime}\right)}{K / J-2 j \pi}
$$

where

$$
J=\left[\int_{0}^{1} d x L(x)\right]^{-1}
$$

We then obtain the admittance from Eq. (2.2) as

$Z_{0} Y(k) \cong 2 \pi k a\left[-\frac{j}{k^{2} \Delta}+\sum_{s=1}^{\infty} \frac{e^{-j b_{s} g / a}}{b_{s}}+j \frac{2 \ln 2}{\pi}\right]$

where we have used the solution of $\mathrm{Eq}$. (3.8) in the Eorm

$$
L(x)=-\frac{x^{-1 / 2}(1-x)^{-1 / 2}}{2 \pi \ln 2}, J=-2 \ln 2 .
$$

\section{Numerical Results and Discussion}

We have used Eq. (3.11) to calculate the real and imaginary parts of the admittance, shown as the solid lines in Figs. $I(a)$ and $1(b)$ for a small pillbox with $b / a=1.1$ and $g / a=.05$. The dots correspond to the results of the compuler program developed lo calculate the longitudinal impedance of an azimuthally symmetric cavity of general shape in a beam pipe. "The agreement between the two confirms the validity of Eq. (3.11).
Furthermore, the "sawtooth" structure of that part of the admittance involving the zeroes of $J_{0}(x)$ depends

primarily on the parameter ka land only logarithmically on $\mathrm{kg}$ ) while the primary dependence on the pillbox size $(b, g)$ is contained only in the smooth part of the susceptance, which is most important for small values of $k^{2} \Delta$.

If one smooths the "sawtooth" structure, equivalent to averaging over $k$ for values of ka above the cutoff of the beam pipe, one finds the features of the broad resonance discussed in depth in an earlier paper.

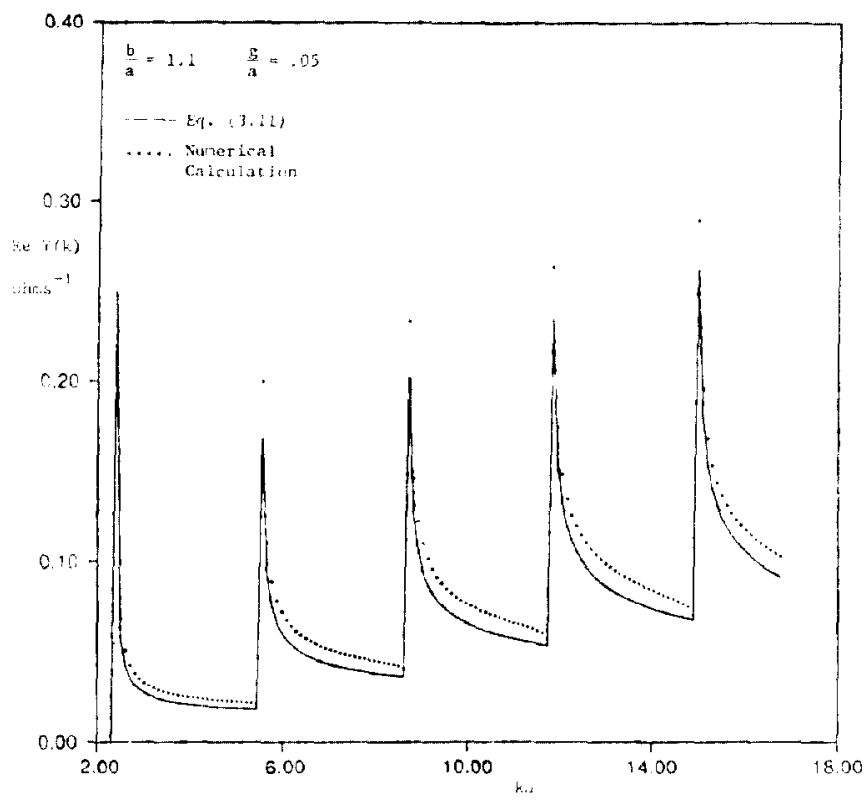

Eigure 1 (a)

Real Part of Admittance as a Eunction of Frequency

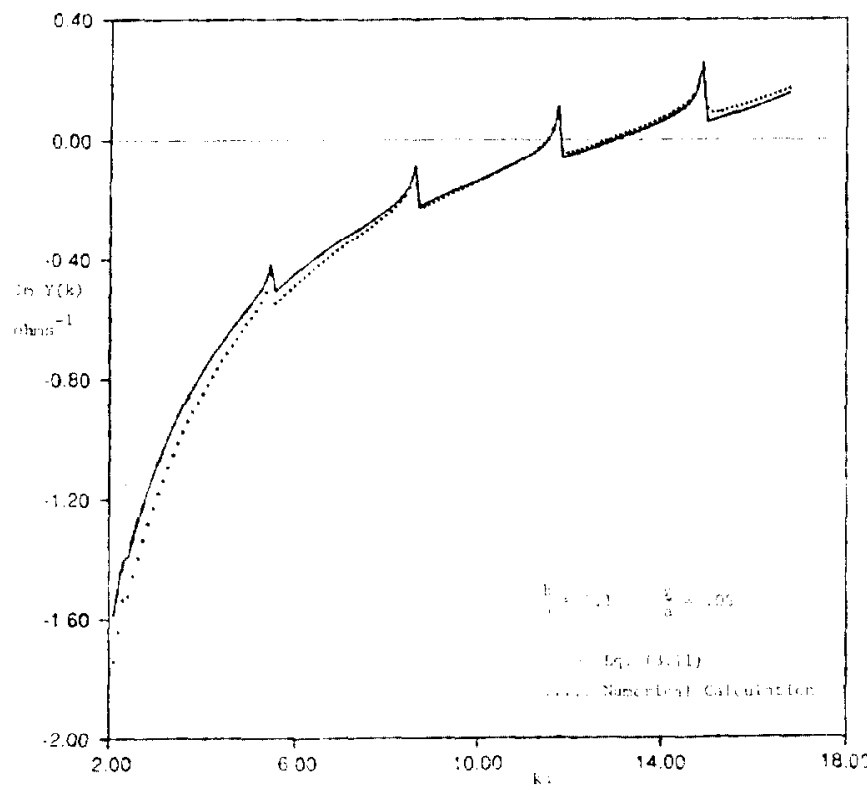

Eigure 1 (b)

Imaginary Part of Admittance as a Function of Erequency 


\section{References}

1. Work supported by the Department of Energy.

2. R.L. Gluckstern and F. Neri, Proceedings of the Particle Accelerator Conference, washington, D.C., March 1987, p. 1069.

3. H. Henke, Eoint Charge Passing a Resonator with Beam Tubes, CERN-LEP-RF/85-41.

4. R.L. Gluckstern, High Frequency Behavior of the Longitudinal Impedance for a Cavity of General Shape, submitted for publication.

5. R.L. Gluckstern and B. Zotter, Coupling Impedance for Asymmetric Cavities, CERN-LEP-613, July 1988.

6. See Appendix 2 .

\section{Appendix A}

In order to demonstrate that

$$
L(x)=-\frac{x^{-1 / 2}(1-x)^{-1 / 2}}{2 \pi \ln 2}
$$

is a solution of $\mathrm{Eq} .(3.8)$, we make the substitutions

$$
x^{\prime}=\frac{1+\cos \theta^{\prime}}{2}, x=\frac{1+\cos \theta}{2}
$$

from which we obtain

$$
\begin{aligned}
& Q \equiv \int_{0}^{1} \frac{d x^{\prime}}{\sqrt{x^{\prime}\left(1-x^{\prime}\right)}} \ln \left|x^{\prime}-x\right| \\
& =\frac{1}{2} \operatorname{Re} \int_{-\pi}^{\pi} d \Theta^{\prime} \ln \left(\frac{\cos \theta-\cos \theta^{\prime}}{2}\right) .
\end{aligned}
$$

Use of the exponential forms for $\cos \theta, \cos \theta^{\prime}$ leads to

$$
\begin{aligned}
Q=\frac{1}{2} \operatorname{Re} \int_{-\pi}^{\pi} d \Theta^{\prime} & {\left[\ln e^{i \theta}+\ln \left(1-e^{-i\left(\Theta^{\prime}+\theta\right)}\right)\right.} \\
& \left.+\ln \left(1-e^{i\left(\theta^{\prime}-\theta\right)}\right)-\ln 4\right] \\
= & -\pi \ln 2+\int_{0}^{\pi} d \psi \ln (1-\cos \psi)
\end{aligned}
$$

where $\psi=\ominus^{\prime} \pm \theta$, and where the last form of $Q$ is clearly independent of $\theta$, as required by Eq. (3.8).

A power series expansion of the integrand in Eq. (A. 3) leads to

$$
Q=-\pi \ln 2+\frac{\pi}{2} \sum_{m=1}^{\infty} \frac{\Gamma\left(m+\frac{1}{2}\right)}{m \Gamma\left(\frac{1}{2}\right) m !} .
$$

The sum over $m$ in Eq. (A.5) can be shown to be equivalent to the sum obtained from the power series expansion of the integrand in

$$
Q=-\pi \ln 2+\frac{\pi}{2} \int_{0}^{1} \frac{d u}{u \sqrt{1-u}}(1-\sqrt{1-u}),
$$

which is readily evaluated as

$Q=-\pi \ln 2+\left.\pi \ln (1+\sqrt{1-u})\right|_{0} ^{1}=-2 \pi \ln 2$

Thus

$$
\int_{0}^{1} L\left(x^{\prime}\right) \ln \left|x^{\prime}-x\right| d x^{\prime}=-\frac{Q}{2 \pi \ln 2}=1 \text {. }
$$

as required in Eq. (3.8), and

$$
J=\left[\int_{0}^{1} d x L(x)\right]^{-1}=-2 \ln 2
$$

as stated in Eq. (3.12) 